\title{
Incidence of intrathyroid location of parathyroid glands and description of their characteristics compared to extrathyroid ones
}

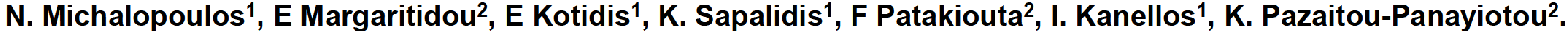

1. 3rd Department of Surgery, Aristotle University of Thessaloniki, AHEPA University Hospital, Thessaloniki, Greece.

2. Department of Endocrinology - Endocrine Oncology, Theagenio Cancer Hospital, Thessaloniki, Greece.

\section{OBJECTIVES}

Total thyroidectomy (TT) is a safe and effective operation. The most frequent complication after TT is hypoparathyroidism. Surgical trauma, devasculization of parathyroid glands and inadvertent parathyroidectomy have been identified as the main causes resulting in postoperative hypocalcaemia. The objectives of this study are:

1. To investigate the incidence of intrathyroid parathyroid glands (ITPGs) in a large cohort 2. To clarify their histological characteristics.

\section{METHODS}

This is a retrospective study of two specialized centers in thyroid diseases. The medical records of patients with thyroidectomy were reviewed. Clinical and demographic characteristics, biochemical results, information regarding operation and histopathological data were routinely collected. Figure 1 show the flow diagram of the study and the exclusion criteria. Patients were divided into two groups: those with parathyroidectomy ( $P$ group) and those without parathyroidectomy (non-P group). According to the location of the dissected parathyroid gland the P-group was sub-divided in ITPGs or extrathyroid parathyroid glands (ETPGs). As ITPG we term the parathyroid gland that is completely surrounding by thyroid tissue or is intracapsular without separate capsule from the thyroid gland Morphological characteristics of ITPGs were compared with the corresponding characteristics of the ETPGs in a case control way.

\begin{tabular}{|c|c|c|c|c|}
\hline Patient characteristics & $\begin{array}{l}\text { P group } \\
(\mathrm{n}=424)\end{array}$ & $\begin{array}{l}\text { Non-P group } \\
(\mathrm{n}=3471)\end{array}$ & $\begin{array}{l}\text { Total } \\
(n=3895)\end{array}$ & $p^{\wedge}$ \\
\hline Age $(y)$, mean [SD] & $52.44[22.61]$ & $53.75[19.6]$ & $53.5[20.57]$ & $\mathrm{n} / \mathrm{s}$ \\
\hline \multirow[t]{2}{*}{ Gender $\mathbf{n}(\%)$} & M $93(22)$ & M 729 (21.1) & M 837(21.49) & $n / s$ \\
\hline & F 331 (78) & F 2742 (78.9) & F $3058(78.51)$ & \\
\hline Operative time (min), mean [SD] & $87.43(13.4)$ & $84.35(15.32)$ & $85.7(14.45)$ & $\mathrm{n} / \mathrm{s}$ \\
\hline Thyroid gland weight (gr), mean [SD] & $45[28.3]$ & $51[43.4]$ & $47[39.8]$ & 0.18 \\
\hline \multicolumn{5}{|l|}{ Diagnosis, $\mathbf{n}(\%)$} \\
\hline Benign thyroid diseases & $275(64,86)$ & $2492(71.8)$ & $2767(71)$ & $\mathrm{n} / \mathrm{s}$ \\
\hline Malignant thyroid diseases & $149(35.14)$ & $979(28.2)$ & $1128(29)$ & 0.04 \\
\hline Transient hypocalcemia n (\%) & $72(17)$ & $520(14.9)$ & $592(15.2)$ & $\mathrm{n} / \mathrm{s}$ \\
\hline Permanemt hypocalcicemia (\%) & $3(0.7)$ & $2(0.06)$ & $5(0.13)$ & 0.001 \\
\hline Hospital stay (days), mean [SD] & $1.5(0.35)$ & $1.4(0.4)$ & $1.4(0.45)$ & $\mathrm{n} / \mathrm{s}$ \\
\hline
\end{tabular}

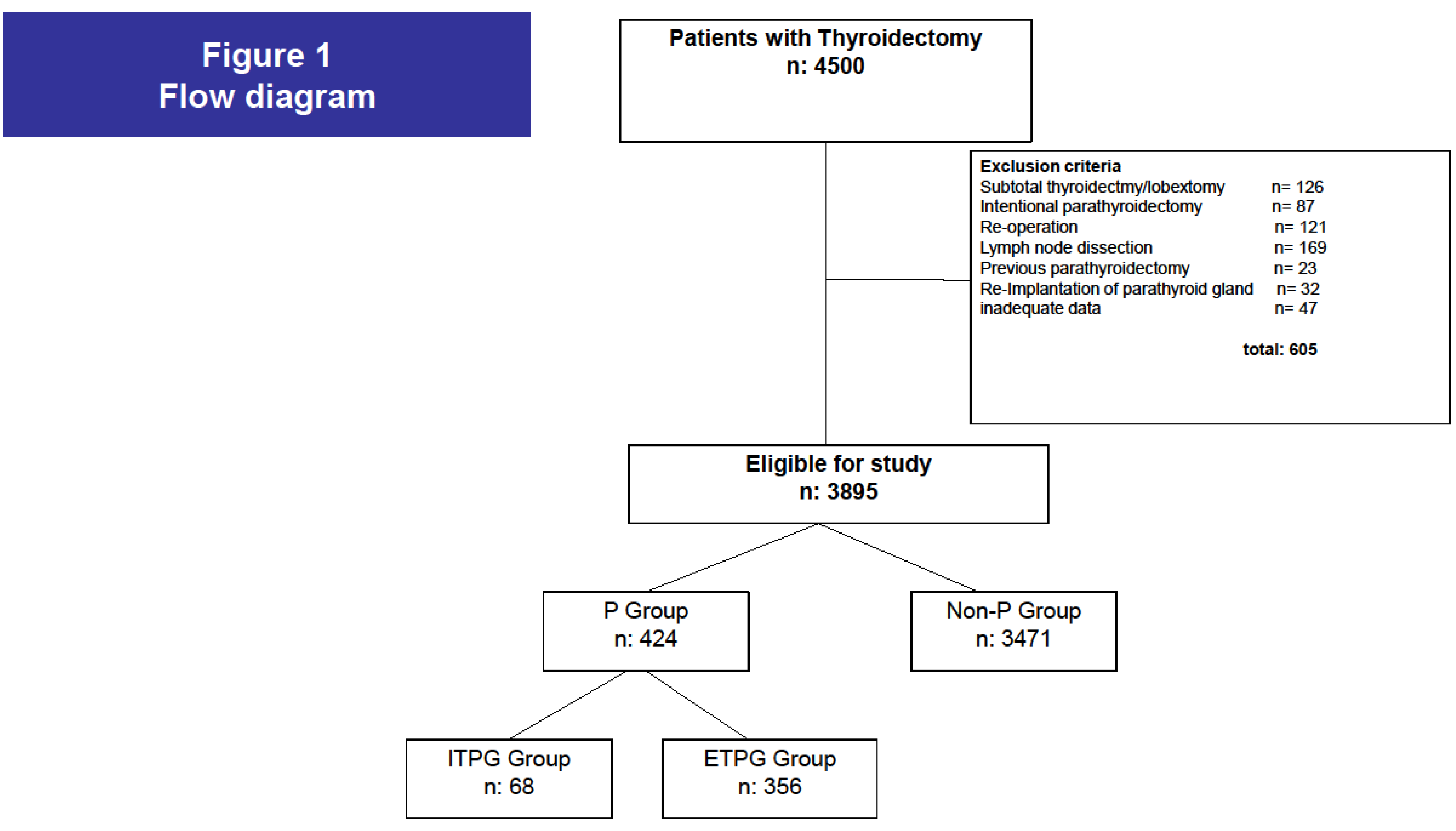

RESULTS

\begin{tabular}{|c|c|c|c|}
\hline Patient characteristics & $\begin{array}{l}\text { ITPG group } \\
(\mathrm{n}=68)\end{array}$ & $\begin{array}{l}\text { ETPG } \\
\text { group } \\
(\mathrm{n}=\mathbf{3 5 6})\end{array}$ & $p^{\wedge}$ \\
\hline Age $(\mathrm{y})$, mean [SD] & 51.9 [21.4] & $52.8[23.1]$ & $\mathrm{n} / \mathrm{s}$ \\
\hline Gender $\mathbf{n}(\%)$ & $\begin{array}{l}\text { M } 15(22) \\
\text { F } 53(78)\end{array}$ & $\begin{array}{l}\text { M } 78 \\
(21.9) \\
\text { F 278 (78.1) }\end{array}$ & $\mathrm{n} / \mathrm{s}$ \\
\hline Operative time (min), mean [SD] & $85.4[18]$ & $88.7[16.5]$ & $\mathrm{n} / \mathrm{s}$ \\
\hline $\begin{array}{l}\text { Thyroid gland weight (gr), mean } \\
\text { [SD] }\end{array}$ & $43[29.8]$ & $46[26.3]$ & $\mathrm{n} / \mathrm{s}$ \\
\hline Diagnosis, $\mathbf{n}(\%)$ & $43(63.24)$ & $231(64.9)$ & $\mathrm{n} / \mathrm{s}$ \\
\hline $\begin{array}{l}\text { Benign thyroid diseases } \\
\text { Malignant thyroid }\end{array}$ & $25(36.76)$ & $125(35.1)$ & \\
\hline $\begin{array}{l}\text { diseases } \\
\text { Number of parathyroid removal } \\
\text { median (range) }\end{array}$ & $1(1-3)$ & $1(1-4)$ & $\mathrm{n} / \mathrm{s}$ \\
\hline Transient hypocalcemia n (\%) & $12(17.6)$ & $60(16.85)$ & $\mathrm{n} / \mathrm{s}$ \\
\hline Permanemt hypocalcicemia (\%) & 1 & 2 & $\mathrm{n} / \mathrm{s}$ \\
\hline Hospital stay (days), mean [SD] & $1.5[0.3]$ & $1.5[0.4]$ & $\mathrm{n} / \mathrm{s}$ \\
\hline
\end{tabular}

\section{CONCLUSIONS}

DThe incidence of ITPGs in our cohort is $1.75 \%$

- Surgical removal of ITPGs does not increase the presence of clinical hypocalcaemia and should be consider a minor complication of TT.

aPatients should be informed prior to TT about the possible presence of ITPGs and, therefore, their probable accidental removal.

\section{References}

bboud B, Sleilaty G, Braidy C, Zeineddine S, Ghorra C, Abadjian G et al. Careful xamination of Thyroid Specimen Intraoperatively to Reduce Incidence of Surg2007;133(11):1105-1110.

1992;164:496-500.

Gourgiotis S, Moustafellos P, Dimopoulos N, Papaxoinis G, Baratsis $S$, Hadjyannakis $E$. Inadvertent parathyroidectomy during thyroid surgery: the cidence of a complication of thyroidectomy. Langenbecks Arch Surg 006;391:557-5 PapadimaA et ark uniniannakis $\mathrm{H}$, Lagoudianakis E, Antonakis $\mathrm{P}$, Genetzakis $\mathrm{M}$ Neck 2008; 30:497-502. Youssef T, Gaballah G, Abd-ElaalE, El-Dosoky E. Assessment of risk factors of 2010;8:207-211

Wang CA. The Anatomic Basis of Parathyroid Surgery. Ann Surg 1976;183:27 